\title{
Super-twisting controllers for wind turbines
}

\author{
C. Tutivén, Y. Vidal, L. Acho and J. Rodellar \\ Universitat Politècnica de Catalunya, \\ Applied Mathematics-III (MA-3) Department, CoDAlab, \\ Comte d'Urgell, 187, 08036, Barcelona, Spain. \\ Phone number:+0034934137309, e-mail: christian.tutiven@upc.edu, yolanda.vidal@upc.edu \\ leonardo.acho@upc.edu,jose.rodellar@upc.edu
}

\begin{abstract}
The main contribution of this paper is to propose new control techniques which not only provide fault tolerance capabilities to the WT system, but also improve the overall performance of the system in both fault free and faulty conditions. Coupled nonlinear aero-hydro-servo-elastic simulations of an offshore wind turbine with jacket platform are carried out. The proposed controllers are based in the super-twisting algorithm (STA) by using feedback of the generator shaft speed as well as the fore-aft and side-to-side acceleration signals of the WT tower.
\end{abstract}

\section{Key words}

fault-tolerant-control, wind turbine control, offshore wind energy, super-twisting

\section{Introduction}

The objective of FTC is to design appropriate controllers such that the resulting closed-loop system can tolerate abnormal operations of specific control components and retain overall system stability with acceptable system performance. Ideally, the closed loop system should be capable of maintaining its pre-specified performance in terms of quality, safety, and stability despite the presence of faults [1]. In general, the FTC approaches can be classified into two types: the passive approach and the active approach. In active schemes, the controller is reconfigured whenever a fault is detected. In passive FTC schemes, the controller's structure is fixed. Because the power industry is used to passive control structures, in this work, we concentrate on this particular scheme.

In previous works (e.g., [2]), it has been proposed the use of classical sliding mode control for WT control. Such approaches deal efficiently with the power regulation objective and provide the advantage of robustness against system uncertainties and perturbations but its well-known drawback has been the discontinuous behavior of the computed control inputs that may derive into a high-frequency oscillation known as chattering. The remarkable properties of the supertwisting algorithm (STA) are: a) accurately regulating and tracking accomplished with finite-time convergence; b) as the control input is a continuous state function, there is a reduction of mechanical stresses and chattering; c) time derivative of the output is not needed; d) robustness with respect to various internal and external disturbances and model uncertainties; e) relatively simple control laws that can be designed based on nonlinear models. In this work, new torque and pitch controllers are proposed based on the STA by introducing the acceleration signals at top tower as a feedback perturbation signal.

In this work, the aero-hydro-servo-elastic simulation tool FAST v8 [3], developed by the National Renewable Energy Laboratory, is used. An offshore 5MW wind turbine benchmark [4] with jacket support is considered as a testbed for the proposed FTC strategies.

\section{Wind turbine description}

A complete description of the wind turbine model can be found in [4] and a detailed descripton of the jacket model is given in[5]. Hereafter, only the generator-converter actuator model, the pitch actuator model and the baseline control strategy are recalled in order to introduce the notation and the concepts employed in following sections.

\section{A. Generator-converter model}

The generator-converter system can be approximated by a first-order differential equation, see [6], which is given by:

$$
\dot{\tau_{r}}(t)+\alpha_{g c} \tau_{r}(t)=\alpha_{g c} \tau_{c}(t),
$$

where $\tau_{r}$ and $\tau_{c}$ are the real generator torque and its reference (given by the controller), respectively. In the numerical simulations, $\alpha_{g c}=50$, see [4]. Moreover, the power produced by the generator, $P_{e}(t)$, may be given by (see [6]):

$$
P_{e}(t)=\eta_{g} \omega_{g}(t) \tau_{r}(t),
$$

where $\eta_{g}$ is the efficiency of the generator and $\omega_{g}$ is the generator speed. In the numerical experiments, $\eta_{g}=0.98$ is used, see [6].

\section{B. Pitch actuator model}

The pitch actuator can be modeled as a second-order linear differential equation with time-dependent variables, pitch angle $\beta(t)$ and its reference $\beta_{c}(t)$ (given by the controller), [6]:

$$
\ddot{\beta}(t)+2 \xi \omega_{n} \dot{\beta}(t)+\omega_{n}^{2} \beta(t)=\omega_{n}^{2} \beta_{c}(t),
$$

where $\omega_{n}$ and $\xi$ are the natural frequency and the damping ratio, respectively. In the numerical experiments, $\xi=0.6$ and $\omega_{n}=11.11 \mathrm{rad} / \mathrm{s}$ are utilized, see [6]. 


\section{Baseline torque and pitch controllers}

The baseline torque and pitch controllers specifications are described in the technical report [4] by the U.S. Department of Energy's National Renewable Energy Laboratory (NREL). Here a brief review of these controllers is given as its performance will be used for comparison with the proposed techniques.

In the full load region of operation, the torque controller maintains constant the generator power; thus,

$$
\tau_{c}(t)=\frac{\mathrm{Pe}_{n}}{\hat{\omega}_{g}(t)},
$$

where $\mathrm{Pe}_{n}$ is the rated power and $\hat{\omega}_{g}$ is the filtered generator speed (see [4]). As the generator may not be able to supply the desired torque depending on the operating conditions, the torque controller is saturated to a maximum of $47,402.9 \mathrm{Nm}$ and a maximum rate limit of $15,000 \mathrm{Nm} / \mathrm{s}$; see [4].

When working in the full load region, a pitch controller is needed to regulate the generator speed. The collective blade pitch gain scheduling PI-controller (GSPI) is used in the literature as a baseline controller to compare the obtained results. This controller was originally developed by Jonkman for the standard land-based 5-MW turbine [4]. The GSPI control has the filtered generator speed, $\hat{\omega}_{g}(t)$, as the input and the pitch servo set-point, $\beta_{r}(t)$, as the output. That is,

$$
\begin{gathered}
\beta_{r}(t)=K_{p}(\theta)\left(\hat{\omega}_{g}(t)-\omega_{g, n}\right)+K_{i}(\theta) \int_{0}^{t}\left(\hat{\omega}_{g}(\tau)-\omega_{g, n}\right) d \tau, \\
K_{p}>0, K_{i}>0
\end{gathered}
$$

where $\omega_{g, n}$ is the nominal generator speed (at which the rated electrical power of the WT is obtained) and the scheduling parameter $\theta$ is taken to be the previously measured collective blade pitch angle. The pitch angle actuators generally present hard constraints on their amplitude and their speed response. Because of this, a pitch limit saturation to a maximum of $45^{\circ}$ and a pitch rate saturation of $8^{\circ} / \mathrm{s}$ are implemented (see [4]) to avoid pitch actuator damage.

\section{Problem statement}

A comprehensive analysis of the STA is conducted, for instance, in [7]. Here, the scalar STA is used to design new torque and pitch controllers. In spite of the coupling existing in WTs, most control strategies for WT uncouple the control problem into different Single Input Single Ouput (SISO) control loops to make easier the control system design. Although the uncoupled assumption, from the control design point of view, these controllers work collaboratively in the over-all closed loop system (see, for example, [8]). In this paper the uncoupled hypothesis for design is used where: a) torque control objectives are to regulate the electrical power and mitigate vibrations in the side-to-side direction and b) pitch control objectives are to regulate the generator speed and mitigate vibrations in the fore-aft direction. WT faults induce vibrations of the corresponding WT subsystems, thus vibration mitigation is an extra control objective for the proposed controllers in order to be able to face with different faulty conditions. Note that both controllers work together to obtain an electrical power regulated to the rated electrical power and, at the same time, a generator speed regulated to its nominal value.

\section{A. Controllers design}

On one hand, we propose the scalar STA-based torque controller

$$
\begin{gathered}
\tau_{c}(t)=-\alpha_{1} \sqrt{\left|P_{e}-\mathrm{Pe}_{n}\right|} \operatorname{sign}\left(P_{e}-\mathrm{Pe}_{n}\right)+y, \\
\dot{y}=-\alpha_{2} \operatorname{sign}\left(P_{e}-\mathrm{Pe}_{n}\right)+\alpha_{3} a_{s s}(t),
\end{gathered}
$$

where $\alpha_{1}, \alpha_{2}, \alpha_{3}>0$ and $a_{s s}(t)$ is the side-to-side acceleration measured at the tower top. Note that we introduce the acceleration as a perturbation signal to give the controller the ability to face with vibrations (and faulty conditions). A stability analysis for this controller is given in the next subsection.

On the other hand, we propose to modify the baseline gain-scheduling pitch controller in the form

$$
\begin{gathered}
\beta_{c}(t)=K_{p}(\theta)\left(\hat{\omega}_{g}(t)-\omega_{g, n}\right)+K_{i}(\theta) z, \\
\dot{z}=\operatorname{sign}\left(\hat{\omega}_{g}(t)-\omega_{g, n}\right)+\alpha_{4} a_{f a}(t),
\end{gathered}
$$

where $\alpha_{4}>0$ and $a_{f a}(t)$ is the fore-aft acceleration measured at the tower top. Note that the acceleration is introduced, similarly to the torque controller, as a perturbation signal. For the proposed pitch controller, as it is a gainscheduling proportional integral control, the controller gains have been heuristically tuned following the same procedure as in [4].

The block diagram in Figure 1 shows the connections between the WT, and the proposed torque and pitch controllers.

\section{B. Torque control stability analysis}

For a perfectly rigid low-speed shaft, a single-mass model for a wind turbine can be considered ([9]),

$$
J_{t} \dot{\omega}_{g}=T_{a}-\tau_{c}
$$

where $J_{t}$ is the turbine total inertia $\left(\mathrm{Kg} \mathrm{m}^{2}\right), \tau_{c}$ is the generator torque $(\mathrm{Nm})$, and $T_{a}$ is the aerodynamic torque (Nm) described as

$$
T_{a}=\frac{1}{2} \rho \pi R^{2} \frac{C_{p}(\lambda, \beta)}{\omega_{r}} u^{3},
$$

where $\rho$ is the air density $\left(\mathrm{kg} / \mathrm{m}^{3}\right), R$ is the rotor radius $(\mathrm{m}), \omega_{r}$ is the rotor speed $(\mathrm{rad} / \mathrm{s}), u$ is the wind speed $(\mathrm{m} / \mathrm{s})$, and $C_{p}(\lambda, \beta)$ is the power coefficient (bounded by the Betz limit). Note that, due to physical constraints, the aerodynamic torque is bounded. Thus, it is realistic to assume that $0<$ $T_{a} \leq \gamma, \forall t \geq 0$.

The STA-based torque control objective is to regulate the electrical power. That is, we define the error:

$$
e(t)=P_{e}(t)-\mathrm{Pe}_{n},
$$




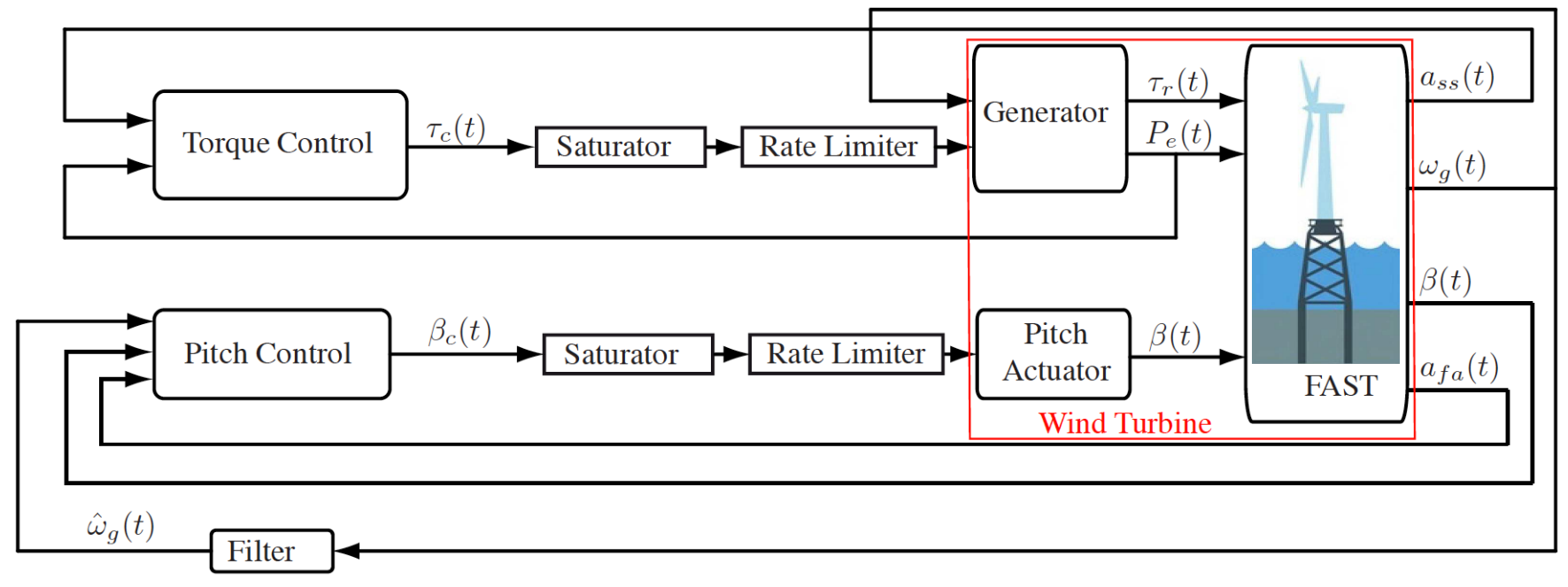

Fig. 1. Block diagram of the closed loop system.

and the control objective is that it converges to zero as time goes on. It is obvious that

$$
\dot{e}(t)=\dot{P}_{e}(t)=\eta_{g}\left[\dot{\omega}_{g}(t) \tau_{r}(t)+\omega_{g}(t) \dot{\tau}_{r}(t)\right] .
$$

Using (1) and (7), from the generator-converter model and WT model respectively, the error dynamics can be written as

$\dot{e}(t)=\eta_{g}\left[J_{t}^{-1}\left(T_{a}-\tau_{c}\right) \tau_{r}(t)+\alpha_{g c} \omega_{g}(t)\left(\tau_{c}(t)-\tau_{r}(t)\right)\right]$,

and, assuming that $\tau_{c}(t)-\tau_{r}(t) \approx 0$, can be simplified to

$$
\dot{e}(t)=\eta_{g} J_{t}^{-1} T_{a} \tau_{c}(t)-\eta_{g} J_{t}^{-1} \tau_{c}^{2}
$$

Finally, linearizing the previous dynamic around $\tau_{c}(t)=0$, the error dynamics yield

$$
\dot{e}(t)=\eta_{g} J_{t}^{-1} T_{a} \tau_{c}(t)
$$

and, as $\eta_{g} J_{t}^{-1} T_{a}$ is positive and bounded, to prove the local stability of this system is equivalent to study the local stability conditions of the system

$$
\dot{e}(t)=\tau_{c}(t)
$$

This system, after substituting (5) gives the closed loop error dynamics,

$$
\begin{aligned}
& \dot{e}(t)=-\alpha_{1} \sqrt{|e|} \operatorname{sign}(e)+y, \\
& \dot{y}=-\alpha_{2} \operatorname{sign}(e)+\alpha_{3} a_{s s}(t) .
\end{aligned}
$$

Since we consider that the side-to-side acceleration, $a_{s s}(t)$, is a perturbation signal (giving the controller the ability to face with vibrations), system (9)-(10) is stable as has been proven in [10]. This finally concludes the stability of the proposed torque control.

\section{Simulation results}

This section presents the performance evaluation of proposed STA controllers. In order to compare between different control systems, the described baseline control system in Section 2-C was used as a frame of reference. Simulations were conducted for a realistic wind speed sequence with mean speed of $14 \mathrm{~m} / \mathrm{s}$, and over $600 \mathrm{~s}$ of run time. This wind speed sequence is illustrated in Fig. 2 with the waves elevation. The rated and cutout wind speeds are $11.4 \mathrm{~m} / \mathrm{s}$ and $25 \mathrm{~m} / \mathrm{s}$, respectively. Thus, the wind profile lies in the above rated region of work.

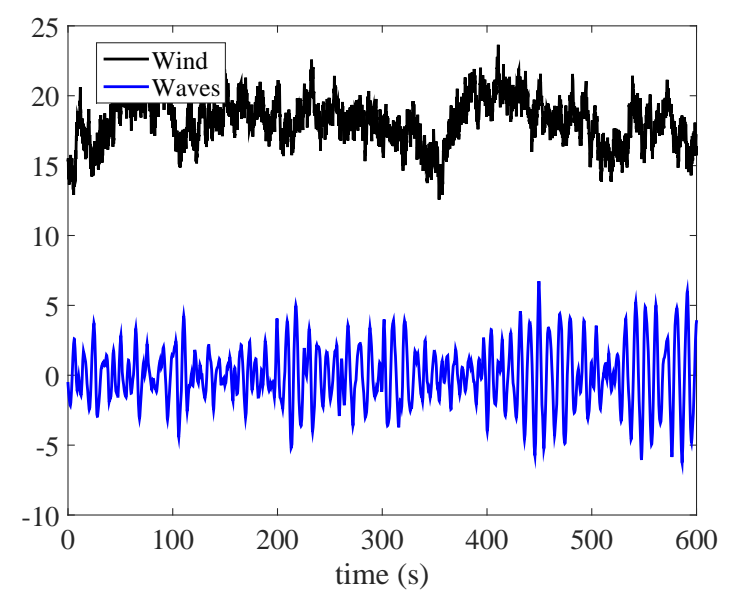

Fig. 2. Wind speed $(\mathrm{m} / \mathrm{s})$ and wave elevation $(\mathrm{m})$.

Here, performance indices are given to present a comparison between STA and baseline controllers:

$$
\begin{gathered}
J_{1}(t)=\int_{0}^{t}\left|a_{f a}(\tau)\right| d \tau,[\mathrm{m} / \mathrm{s}] \\
J_{2}(t)=\int_{0}^{t}\left|a_{s s}(\tau)\right| d \tau,[\mathrm{m} / \mathrm{s}] \\
J_{P}(t)=\int_{0}^{t}\left|P_{e}(\tau)-\mathrm{Pe}_{n}\right| d \tau,[J]
\end{gathered}
$$


where $a_{f a}(t)$ and $a_{s s}(t)$ are the fore-aft and the side-to-side accelerations, respectively, at the tower top.

\section{A. Healthy}

First, the high performance of the STA controllers is demonstrated in fault-free operation of the wind turbine.
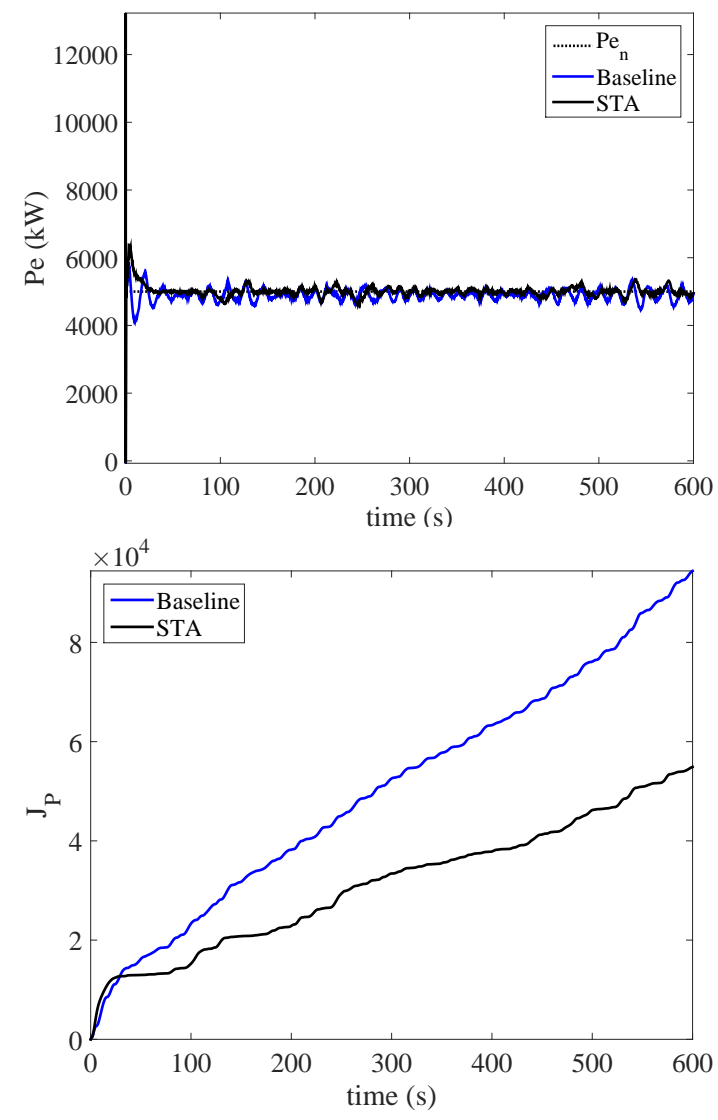

Fig. 3. Electrical power (top) and $J_{P}$ index (bottom).

The proposed controllers improve the power generation quality as can be seen in Figure 3. Due to the rate-limiter action and the complexity of the WT model used for simulation (FAST), the finite-time convergence behavior of the STA torque controller is not evidenced in the results, as can be seen in Figure 3 (top). The $J_{P}$ performance index is improved, that is the error in the regulation of the electrical power is reduced. In a 600 seconds simulation, the accumulated error is almost halved with respect to the baseline strategy as can be seen in Figure 3 (bottom).

The generator speed has higher oscillations for the baseline controllers as shown in Figure 4 (top). The proposed STA does not induce increased mechanical stress as there are no strong torque variations, as can be seen in Figure 4 (bottom). The torque generator remains smooth and tracks more efficiently the wind fluctuations than in standard control. Indeed, and as expected, this leads to a reduction of the accelerations in the tower, as can be seen in Figure 5. It is noteworthy that the accelerations in the fore-aft direction have been dramatically improved whereas accelerations in
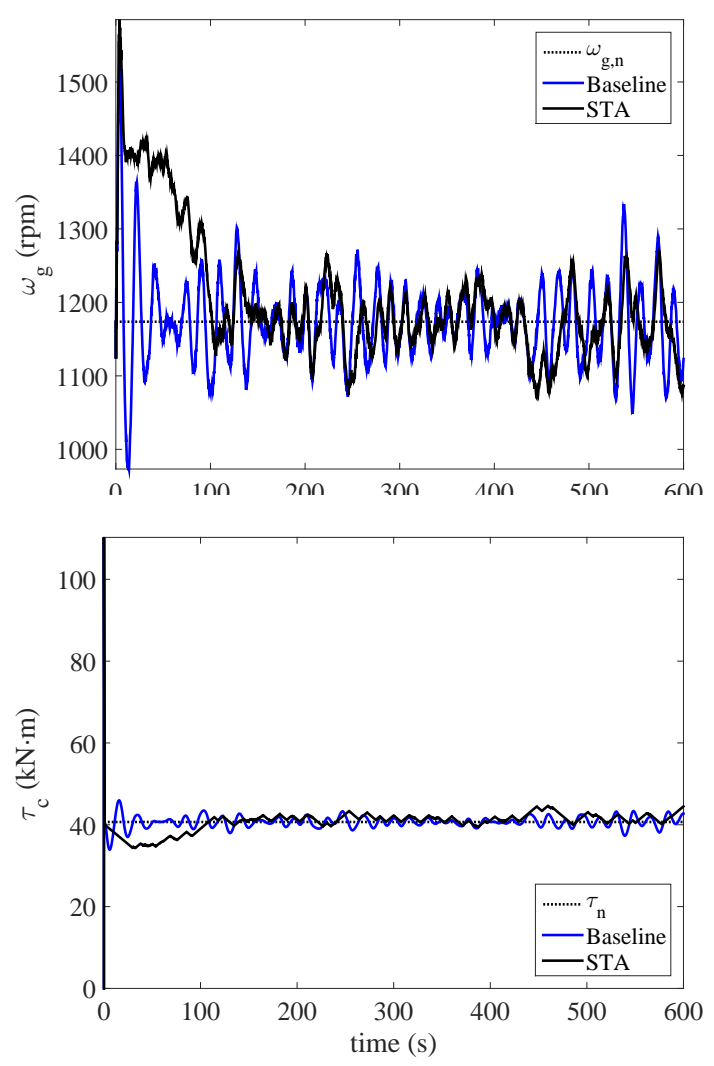

Fig. 4. Generator speed (top) and torque control (bottom).

the side-to-side direction are comparable to the ones obtained with the baseline control.

The platform rotational and translational data is shown in Fig. 6. A reduction is obtained in the pitch tilt angle and the horizontal surge displacement with the proposed STA, with comparable results in the roll tilt and yaw angles and the horizontal sway and heave displacements with respect to the baseline controllers.

Recall that, when designing the pitch angle control loop, it is of great importance to avoid a high activity of the pitch, since it could not only damage the pitch actuators but also give rise to unstable modes of operation, see, for instance, [11]. The pitch control, shown in Figure 7, is smoothed with the STA-based controllers. This lower pitch activity leads to lower mechanical stress (vibration mitigation) spreading the wind turbine lifetime and also resulting in softer output power.

Remark 1. The gains $\alpha_{1}=0.1, \alpha_{2}=200, \alpha_{3}=$ 1 , and $\alpha_{4}=5$ are used in the simulations. They were selected in order to reduce the fore-aft motion. However, other gain values could be used, for example, to obtain also an improvement in the side-to-side direction.

\section{B. Pump wear of pitch actuator}

This fault changes the dynamics of the pitch actuator. A detailed description can be found in [6].

The simulation results for this faulty case show that:

- The transient response of the electrical power has a 

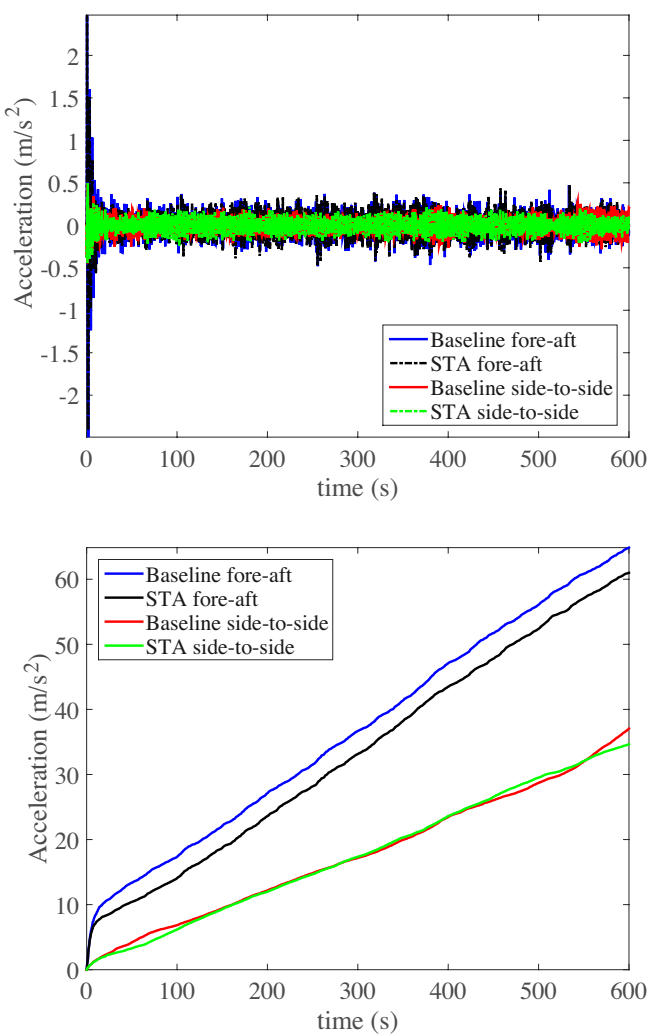

Fig. 5. Fore-aft and side-to-side accelerations (top) and related indices (bottom) at the tower top.

larger oscillation for the baseline controller, see Fig. 8.

- Similarly to the healthy case, the generator speed for the baseline controller has larger oscillations.

- The accelerations at tower top are improved in the fore-aft direction and comparable in the side-to-side direction, see Fig. 9.

- Similarly to the healthy case, a reduction is obtained in the pitch tilt angle and the horizontal surge displacement with the proposed STA, with comparable results in the roll tilt and yaw angles and the horizontal sway and heave displacements with respect to the baseline controllers.

- The blade pitch angle is always within the authorized variation domain, as shown in Fig. 10, but with higher oscillations for the baseline controller. Thus, our proposed controller induces less vibrations in the structure as the range of movement of the pitch angle is smaller.

\section{Conclusions}

This paper addressed the design of a robust STA for efficient and reliable control of a large off-shore wind turbine with jacket platform operating in the full load region. Compared to the baseline controllers, the developed STAcontrollers have been able to improve the overall performance of the wind turbine in healthy and faulty conditions,
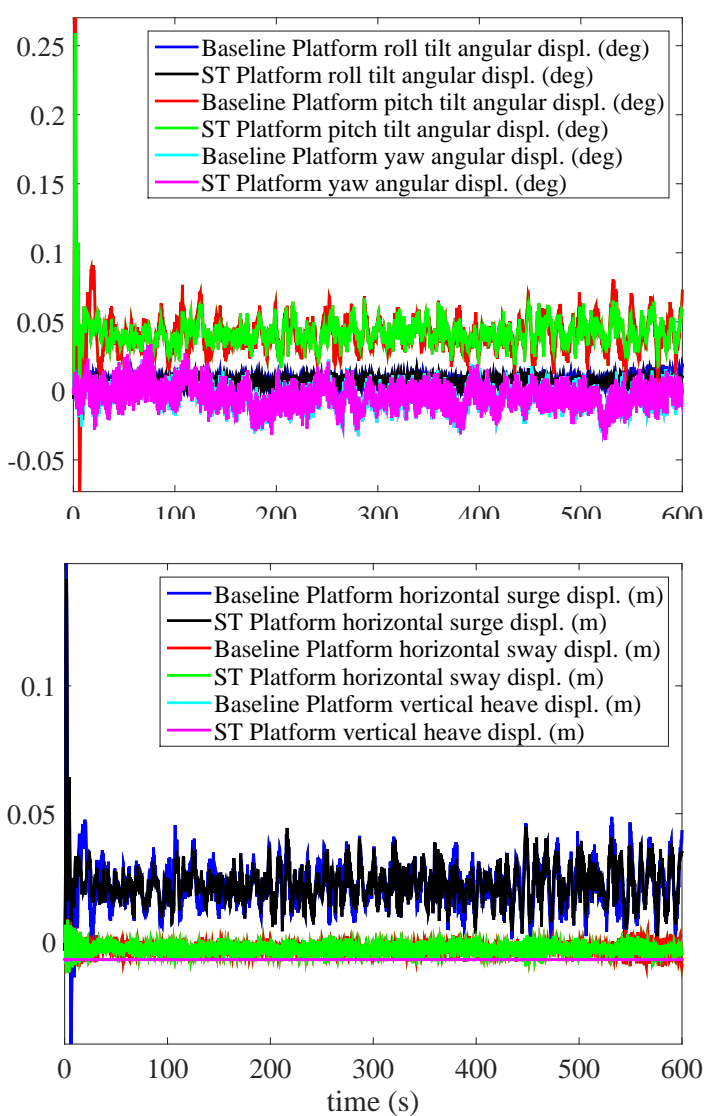

Fig. 6. Platform rotational data (top) and platform translational data (bottom).

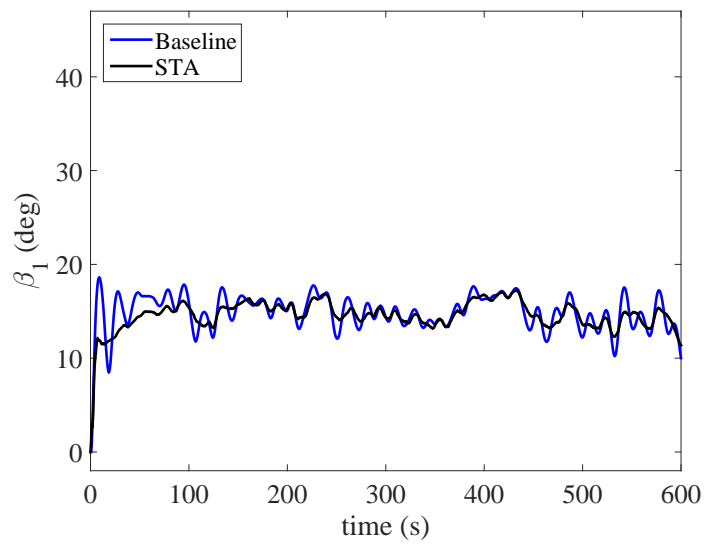

Fig. 7. Pitch angle.

and to reduce the fore-aft and side-to-side accelerations with respect to the baseline control.

\section{Acknowledgments}

This work has been partially funded by the Spanish Ministry of Economy and Competitiveness through the research projects DPI2014-58427-C2-1-R, and DPI2015$64170-\mathrm{R}$, and by the Catalonia Government through the research project 2014 SGR 859. 

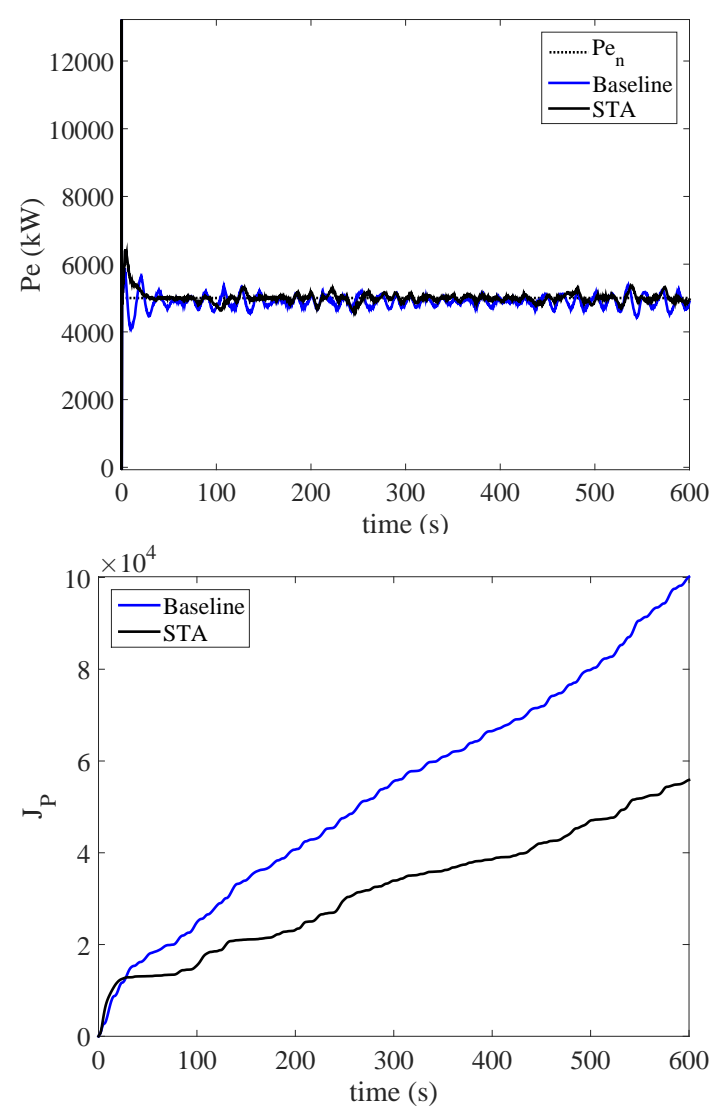

Fig. 8. Electrical power (top) and $J_{P}$ index (bottom) under pump wear faulty condition.

\section{References}

[1] R. J. Patton, Fault-tolerant control systems: The 1997 situation, in: IFAC symposium on fault detection supervision and safety for technical processes, Vol. 3, 1997, pp. 1033-1054.

[2] O. Barambones, Sliding mode control strategy for wind turbine power maximization, Energies 5 (7) (2012) 2310-2330.

[3] N. Kelley, B. Jonkman, NWTC information portal (fast v8) (Last modified 31-March-2015)

URL https://nwtc.nrel.gov/FAST8

[4] J. M. Jonkman, S. Butterfield, W. Musial, G. Scott, Definition of a 5-MW reference wind turbine for offshore system development, Tech. rep., National Renewable Energy Laboratory, Golden, Colorado, nREL/TP-500-38060 (2009).

[5] F. Vorpahl, W. Popko, D. Kaufer, Description of a basic model of the" upwind reference jacket" for code comparison in the oc4 project under iea wind annex 30, Fraunhofer Institute for Wind Energy and Energy System Technology IWES

[6] P. Odgaard, K. Johnson, Wind turbine fault diagnosis and fault tolerant control - an enhanced benchmark challenge, in: Proc. of the 2013 American Control Conference-ACC,(Washington DC, USA), 2013, pp. $1-6$.

[7] V. Utkin, On convergence time and disturbance rejection of supertwisting control, Automatic Control, IEEE Transactions on 58 (8).

[8] A. Diaz de Corcuera, A. Pujana-Arrese, J. M. Ezquerra, A. Milo, J. Landaluze, Design of robust controllers for load reduction in wind turbines, in: N. Luo, Y. Vidal, L. Acho (Eds.), Wind Turbine Control and Monitoring, Advances in Industrial Control, Springer International Publishing, 2014, pp. 97-133.

[9] B. Boukhezzar, L. Lupu, H. Siguerdidjane, M. Hand, Multivariable control strategy for variable speed, variable pitch wind turbines, Renewable Energy 32 (8) (2007) 1273 - 1287

[10] J. A. Moreno, On strict lyapunov functions for some non-homogeneous super-twisting algorithms, Journal of the Franklin Institute 351 (4) (2014) 1902-1919.
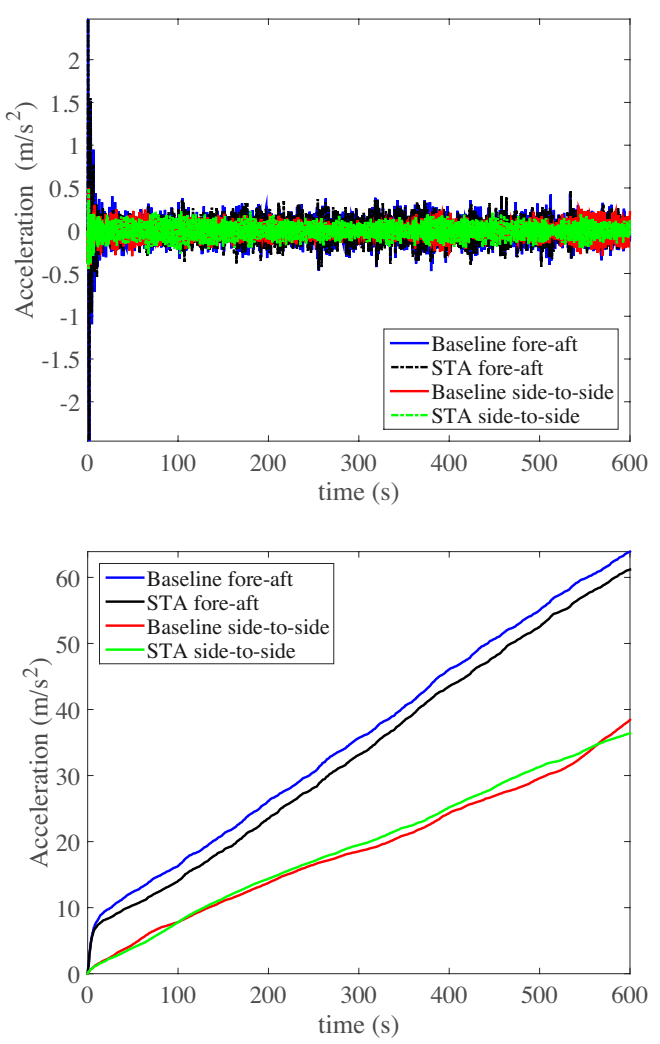

Fig. 9. Fore-aft and side-to-side accelerations (top) and related indices (bottom) at the tower top under pump wear faulty condition.

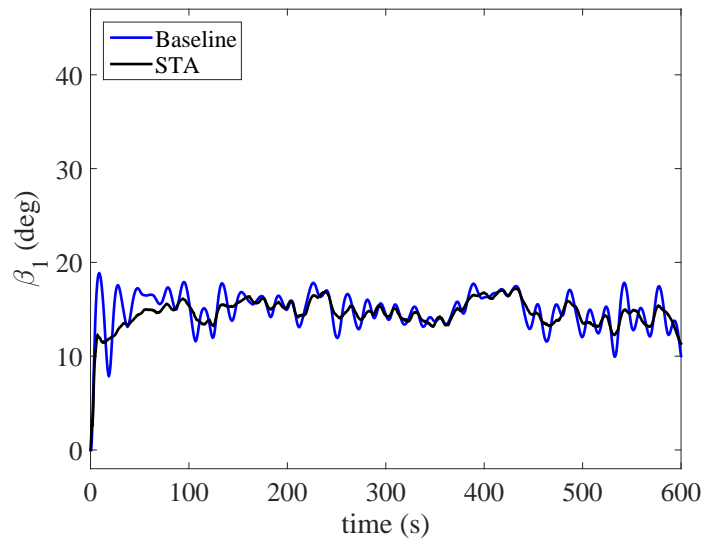

Fig. 10. Pitch angle under pump wear faulty condition.

[11] F. D. Bianchi, H. De Battista, R. J. Mantz, Wind turbine control systems: principles, modelling and gain scheduling design, Springer Science \& Business Media, 2006. 